\title{
Caracterización de los sistemas de producción de ganado bovino en las cuencas ganaderas de Ventilla, Florida y Leyva -región Amazonas
}

\section{Characterization of cattle production systems in the livestock basins of Ventilla, Florida and Leyva-Amazonas region}

\author{
Luis Murga $^{1}$, Héctor Vásquez ${ }^{2}$, Joseph Bardales ${ }^{3}$
}

\section{RESUMEN}

Con el objetivo de caracterizar los sistemas de producción de bovinos en las cuencas ganaderas de Molinopampa y Olleros en la provincia de Chachapoyas, Yambrasbamba y Florida en la provincia de Bongará, Huambo y Limabamba en la provincia de Rodríguez de Mendoza - región Amazonas. Se aplicó una encuesta a 143 ganaderos, comprendiendo los rubros sociales, económicos, técnicos y de producción. Los datos se ordenaron en una matriz de datos y se procesaron en software SPSS versión 17, mediante estadística descriptiva. Los resultados evidenciaron que la mayor parte de los productores fueron partidarios del manejo con limitada tecnología, y solo una minoría opta por técnicas medianamente actualizadas e incompletas. De los encuestados, $36 \%$ tiene título de propiedad, $18 \%$ utilizan registros de producción, presentando un sistema semi-intensivo de crianza, con alimentación a base de pastos de corte, pastos mejorados y algunos ganaderos utilizan otros alimentos terminados, con limitada infraestructura ganadera. La comercialización de la leche es a través de la empresa Gloria, microempresas y mercados informales. En general, se encontró debilidades como la falta de infraestructura y equipo adecuado, por lo que es necesario la intervención de los actores del desarrollo agropecuario, para mejorar la actividad ganadera y hacerla más competitiva.

Palabras clave: Caracterización, cuencas ganaderas, sistema de producción, pastos mejorados, desarrollo agropecuario.

\begin{abstract}
With the objective of characterizing cattle production systems in the livestock basins of Molinopampa and Olleros in the province of Chachapoyas, Yambrasbamba and Florida in the province of Bongará, Huambo and Limabamba in the province of Rodríguez de Mendoza - Amazonas region. A survey was applied to 143 breeders, including social, economic, technical and production items. The data was ordered in a data matrix and processed in software SPSS version 17, using descriptive statistics. The results showed that most of the producers were supporters of management with limited technology, and only a minority opt for moderately updated and incomplete techniques. Of the respondents, $36 \%$ have property title, $18 \%$ use production records, presenting a semi-intensive farming system, with feed based on cutting pastures, improved pastures and some livestock farmers use other finished foods, with limited livestock infrastructure. The marketing of milk is through the company Gloria, microenterprises and informal markets. In general, weaknesses were found such as the lack of adequate infrastructure and equipment, which is why the intervention of agricultural development actors is necessary to improve livestock activity and make it more competitive.
\end{abstract}

Keywords: Characterization, livestock basins, production system, improved pastures, agricultural development.

Investigador del IGBI y docente FIZAB-UNTRM.

${ }^{2}$ Investigador del IGBI-UNTRM 


\section{INTRODUCCIÓN}

La ganadería bovina constituye una actividad productiva de significativa importancia para el desarrollo económico de la región Amazonas con aportes sustanciales en la producción de leche y carne para esta región del Perú. En la región Amazonas se han registrado 69562 unidades agropecuarias ocupando 1766278,65 hectáreas (ha), de las cuales 53275,97 ha son pastos cultivados. Por otra parte, en los distritos en estudio se encuentra el $19,89 \%$ de la población de ganado de la región y el $71 \%$ de los ganaderos se dedican a la producción de leche (INEI, 2012).

La producción de ganado bovino en la región Amazonas, se desarrolla bajo sistemas netamente pastoriles con bajos niveles de suplementación. Las características agroclimáticas de la región determinan diferentes opciones de utilización del recurso tierra que se destina a distintas actividades ganaderas y agrícolas, y la base pastoril destinada a la producción de leche y carne tiene que competir por la tierra con las otras actividades agrarias (Vásquez, 2016).

En un sistema ocurren un sinnúmero de fenómenos complejos que requieren una evaluación desde el punto de vista holístico, que permitan conocer mejor cada uno de los componentes del sistema al igual que sus relaciones entre ellos. Una herramienta utilizada para la evaluación de un sistema, lo constituye la caracterización la cual no es más que la descripción de las características principales y múltiples interrelacionadas de las organizaciones (Bolaños, 1999).

Entre las características que se pueden evaluar en los sistemas ganaderos bovinos de los distritos de Molinopampa y Olleros en la provincia de Chachapoyas; Florida y Yambrasbamba en la provincia de Bongará; Huambo y Limabamba en la provincia de Rodríguez de Mendoza son: datos productivos, identificación de razas y cruces, manejo sanitario y reproductivos, con el objetivo de caracterizar los sistemas de producción de ganado bovino, en base a aspectos sociales, técnicos y económicos; de las principales cuencas antes mencionadas, de la región Amazonas.

\section{MATERIAL Y MÉTODO}

\section{Localización}

El estudio se desarrolló en seis cuencas ganaderas, siendo Molinopampa y Olleros en la provincia de Chachapoyas; Florida y Progreso en la provincia de Bongará, Huambo y Limabamba en la provincia de Rodríguez de Mendoza.

\section{Muestreo de productores}

La unidad de muestreo estuvo constituida por los productores de ganado bovino de las cuencas ganaderas antes mencionadas. La muestra fue probabilística, utilizando muestreo aleatorio estratificado, proporcional a los tamaños de cada cuenca. La selección de la muestra se realizó de forma aleatoria para cada cuenca. El tamaño de la muestra de miembros seleccionados se obtuvo empleando la fórmula propuesta por Cochran (1998).

$$
n_{0}=\frac{\left(\sum W_{h} \sqrt{p_{h} q_{h}}\right)^{2}}{V}
$$

Dónde: Wh es la ponderación en cada estrato, ph es la proporción de miembros que poseen la característica de interés en el cuestionario, qh es la proporción de miembros que no poseen la característica de interés en el cuestionario y $\mathrm{V}$ es la varianza esperada, y calculada por la siguiente fórmula:

$$
V=(d / Z)^{2}
$$

Dónde "d" es la precisión (error máximo de estimación) y "Z" es el valor de la normal asociado al nivel de confianza. $\operatorname{Si~}_{0} \mathrm{~N}<5 \%$, entonces $\mathrm{n}=\mathrm{n}_{0}$

$$
\text { Si } \mathrm{n}_{0 /} \mathrm{N} \geq 5 \% \text {, entonces } n=\frac{n o}{1+\frac{\sum W h * p h * q h}{N V}}
$$

La asignación de la muestra fue con afijación proporcional al tamaño del estrato (las cuencas ganaderas de Molinopampa y Olleros en la provincia de Chachapoyas; Florida y Progreso en la provincia de Bongará, Huambo y Limabamba en la provincia de Rodríguez de Mendoza) (Tabla 1). 
Tabla 1. Distribución de la muestra en cada zona de interés.

\begin{tabular}{lccccccc}
\hline Estratos & $\mathbf{N h}^{\mathbf{1}}$ & $\mathbf{W}_{\mathbf{h}}$ & $\mathbf{n h}_{\mathbf{h}}$ & $\mathbf{p h}$ & $\mathbf{\mathbf { q } _ { \mathbf { h } }}$ & $\mathbf{W}_{\mathbf{h} \mathbf{p h}} \mathbf{q} \mathbf{h}$ & $\mathbf{W h}^{*}\left(\mathbf{p}_{\mathbf{h}} \mathbf{g}_{\mathbf{h}}\right)^{\wedge} \mathbf{0 . 5}$ \\
\hline Molinopampa & 516 & 0.17 & 24 & 0.5 & 0.5 & 0.043 & 0.08535 \\
Olleros & 80 & 0.03 & 4 & 0.5 & 0.5 & 0.007 & 0.01323 \\
Florida (Pomacochas) & 595 & 0.2 & 28 & 0.5 & 0.5 & 0.049 & 0.09841 \\
Yambrasbamba (Progreso) & 504 & 0.17 & 24 & 0.5 & 0.5 & 0.042 & 0.08336 \\
Huambo & 946 & 0.31 & 45 & 0.5 & 0.5 & 0.078 & 0.15647 \\
Limabamba & 382 & 0.13 & 18 & 0.5 & 0.5 & 0.032 & 0.06318 \\
& $\mathbf{3 0 2 3}$ & $\mathbf{1 . 0 1}$ & $\mathbf{1 4 3}$ & $\mathbf{3 . 0}$ & $\mathbf{3 . 0}$ & $\mathbf{0 . 2 5 1}$ & $\mathbf{0 . 5 0 0 0 0}$ \\
\hline
\end{tabular}

$\mathrm{nh}=\mathrm{Wh}(\mathrm{n})$, donde $\mathrm{h}=1,2,3,4,5$ y $6 . \mathrm{Y}$ si “d” $=0.08$ y el coeficiente de confianza del 95\%, entonces " $\mathrm{Z}$ " será equivalente a 1.96, dando como valor de "V" a 0.001665973 . Óptimo supuesto de $\mathrm{N}^{\circ}=150 \mathrm{y}$ factor de corrección $\operatorname{de} \mathrm{N}=143$.

Se estableció que el tamaño de la muestra es de 143 productores agropecuarios que participaron directamente en el estudio, a los cuales se aplicó la encuesta según la metodología de Ruiz et al. (2012). En la que se realizó una serie de preguntas al productor sobre: el título de propiedad, acceso al predio y manejo de pastizales y bosques. Tipo de cultivo agrícola sembrado, instalaciones ganaderas, prácticas de pastoreo, suministro de agua, alimentación, registros y sanidad animal. Calidad y venta de leche, métodos de reproducción del ganado bovino, razas bovinas y categoría de ganado y valor económico del hato.

\section{Análisis de datos}

El análisis de datos se realizó usando estadística descriptiva (tablas de contingencia) en el software estadístico SPSS versión 17.0., para Windows.

\section{RESULTADOS}

Título de propiedad, acceso al predio y manejo de pastizales y bosques

En las zonas en estudio, la ganadería bovina se caracteriza por manejar principalmente el sistema extensivo. De la población en estudio se tiene que el $34,3 \%$ poseen título de propiedad de sus predios, el $69,9 \%$ de los ganaderos tiene acceso a su propiedad por camino de herradura, el $36,4 \%$ cultivan pasto de corte, el $62.2 \%$ poseen pastos naturales y el $94.4 \%$ no aplica fertilizantes a sus potreros. Además, $62,9 \%$ de los productores conservan bosques en su fundo. Se encontró que la extensión del fundo donde se dedican a la crianza de ganado bovino tuvo un máximo de 200 hectáreas (ha) con una media de 22,67 ha, hectáreas de pasturas con que cuentan los ganaderos oscilo entre 1 a 83 ha, con una media de $11,86 \pm 12,37$; de las cuales, pasto de corte como máximo es 30 ha, con una media de 2,86 $\pm 6,33$; pasto mejorado con máximo de 60 ha, con una media de $5,10 \pm 8,84$; pasto natural de 50 ha con una media de 4,52 $\pm 8,62$ y de bosque que aún posee en su fundo osciló entre 0 a 200 ha con una media de $9,37 \pm 22,76$.

\section{Cultivos agrícolas}

En general el produ

ctor ganadero aparte de la actividad ganadera, también se dedica a otras actividades como: el 25,9\% cultivan café, el 7,0\% maíz, el 4,2\% papa y el $15,4 \%$ se dedican a otros cultivos. El cultivo que más predomina en la zona de Florida, Olleros y Molinopampa es papa $(14 \% ; 50 \%$ y 4,2\%) respectivamente, en Yambrasbamba, Huambo, Limabamba, la actividad que más se da es el café $(16,7 \% ; 51,1 \% ; 10 \%)$ respectivamente.

\section{Instalaciones ganaderas}

De los ganaderos evaluados sólo el 12,6\% cuentan con corral de manejo, el 14,7\% tienen manga, el $25,9 \%$ tienen corral de terneros y el 14,0\% cuenta con sala de ordeño. Florida, Molinopampa, Limabamba y Olleros son las zonas en que más del $15 \%$ de los ganaderos cuentan con corral de manejo, manga. Además de los mencionados, Yambrasbamba también está sobre el $15 \%$ en que los ganaderos tienen corrales para terneros. Solo se encontró en dos zonas a productores que tienen sala de ordeño por sobre del $20 \%$ de la población ganadera, siendo esta Florida y Molinopampa. Las otras zonas estudiadas si cuentan, pero son escaso los productores respecto al total por zona. 


\section{Prácticas de pastoreo}

La actividad ganadera necesita ser monitoreada, debido a que los animales pueden cruzar los límites de pastoreo. En general de las zonas evaluadas, el $36,4 \%$ de los ganaderos poseen cercos vivos, el $86,7 \%$ tiene cerco de alambre y el $16,8 \%$ utiliza cerco eléctrico. Independientemente cada zona usa un tipo de división de su potrero, encontrándose en todas las zonas el uso de cerco eléctrico usado más del $80 \%$ por los ganaderos.

\section{Suministro de agua}

En general el $45,5 \%$ de los ganaderos poseen abrevaderos en sus potreros para el suministro de agua a los animales, el 38,5\% de los ganaderos manifestó que proporciona una vez al día, el 11,0\% entre 2 a 3 veces al día y el 50,3\% proporciona agua ad libitum. El suministro de agua ad libitum es la practica más común en todas las zonas, encontrándose desde el 30\% hasta el 100\% de los ganaderos el uso de esta práctica.

\section{Alimentación, registros y sanidad animal}

Los ganaderos de las zonas evaluadas, el 85,3\% de los ganaderos alimenta a su ganado sólo con pasto, $\mathrm{y}$ el 14,7\% utiliza otro producto además del pasto. Además, el 97,9\% de los ganaderos proporcionan sales minerales; de los cuales, el $16 \%$ les proporciona diariamente, el 49,7\% semanal, el 18,2\% quincenal, el $4,9 \%$ de forma mensual y la diferencia $(9.1 \%)$ no lo hace. En general, solo el 18,2\% de los ganaderos utilizan registros de producción, siendo Florida y Olleros los que más usan registros (con rango de $21.4 \%$ hasta $25,0 \%$ ) respecto a los otros lugares. Con respecto a la sanidad animal, el estudio mostró que el $32,9 \%$ de los ganaderos utilizan calendario sanitario; mientras que el $32,2 \%$ de los ganaderos entrevistados respondió que aplican la bacteriana triple de forma semestral, el $15 \%$ de forma anual y el $52 \%$ no aplican la bacteriana triple. La desparasitación, el 7,7\% contestó que lo realiza de forma bimestral, el 68,5\% trimestral, el $16 \%$ semestral y el 5,6\% anual.

En las zonas de estudio, el suministro de vitaminas y minerales se realiza de la siguiente forma: el 31,5\% de los ganaderos aplica de manera trimestral, el 14\% mensual, el $23 \%$ semestral y el $14 \%$ anual. A la pregunta cada que tiempo realizan descarte de mastitis, el $11 \%$ respondió todos los días, el $2 \%$ anual, el $11 \%$ semestral y el $68,5 \%$ nunca realizan descarte de mastitis. Con respecto al descarte de brucelosis y tuberculosis, el 32,2\% de la población en estudio si realiza el descarte, mientras que el $67,8 \%$ no realiza descarte de estas enfermedades. E1 96\% de los ganaderos saben aplicar inyecciones al ganado; siendo el $42 \%$ que aplican intramuscular, el $24 \%$ subcutánea y el 30\% saben aplicar ambas formas de aplicación de inyectables.

\section{Calidad y venta de leche}

De acuerdo al estudio, en relación a la calidad de leche, el 25,2\% de los ganaderos realizan análisis bromatológico de la leche. Las zonas con más estudios en análisis de bromatológica son Molinopampa (91.7\%) y Olleros (75\%), las otras zonas están por debajo del $25 \%$ de la población. Los ganaderos en general, el 8,4\% vende a la empresa Gloria, el $11.2 \%$ vende a empresa agroindustrial y el $80,4 \%$ vende a otros mercados. La produccion y comercializacion de leche de los distritos en estudio, va desde 0 a 200 litros de leche al dia, con una media de $31,67 \pm 68.67$; todo este volumen de leche se oferta al mercado, sobre el precio de venta de leche oscila entre 0,6 a 2,5 soles, dependiendo de la calidad bromatológica.

\section{Métodos de reproducción}

Los ganaderos indican que 67,8\% utilizan monta natural como método reproductivo, el 9,8\% utilizan inseminación artificial, y el 16,8\% utilizan ambos métodos reproductivos; Además, solo $5.6 \%$ de ganaderos Molinopampa utiliza transferencia de embriones.

\section{Razas bovinas y categoría de ganado}

En las zonas evaluadas en general se encontraron raza Brown Swiss, Simmental, Holstein y criollo. De las cuales 83 cabezas de Brown Swiss, con media de $15,06 \pm 19,71 ; 0$ a 54 cabezas Simmental, con media de 2,56 $\pm 7,08 ; 0$ a 24 cabezas Holstein, con media de $1,06 \pm 3,76 ; 0$ a 60 cabezas Criollo, con media de $1,14 \pm 6,30$; también se encontró que existen cruces como Brown Swiss x Simmental con un rango entre 0 a 75 cabezas; cruce de Brown Swiss x Holstein en un rango entre 0 a 25 cabezas, cruce de Holstein x Gyr con un máximo de 13 cabezas.

Sobre la categorización del ganado bovino, se encontró que en los distritos en estudio el hato fluctúa entre: 0 a 38 toros mayores de un año, 0 a 18 toretes de siete meses a un año y medio, 0 a 27 vacas en ordeño; 0 a 25 vaquillonas, 0 a 18 vaquillas de 7 meses a un año y medio y 0 a 15 terneros y terneras.

\section{Valor económico del hato}

El valor económico en soles del ganado bovino según categorías en los distritos en estudio fue: toro mayor a 
1,5 años fluctúa entre 155 a 10000 soles; torete de 7 meses a 1,5 años oscila entre 200 a 5000 soles; vaca en ordeño oscila entre 400 a 7000 soles; vaca seca estuvo en el intervalo de 200 a 8000 soles; vaquillona preñada fluctúo entre 800 a 9000 soles; vaquilla de 7 meses a 1,5 años varía entre 100 a 4000 soles; ternera de 0 a 6 meses varía entre 100 a 3000 soles; ternero de 0 a 6 meses varía entre 100 a 2000 soles, esto varía de acuerdo a la raza y edad.

\section{DISCUSIÓN}

\section{Título de propiedad, acceso al predio y manejo de pastizales y bosques}

En la historia agraria del Perú hay antecedentes que han regulado el límite de las tierras. Es así que durante el segundo gobierno del presidente Manuel Prado (1956 -1962) se nombró una Comisión para la Reforma Agraria y la Vivienda, conformada por hacendados, una de las conclusiones de la comisión fue que en la costa el tamaño de las propiedades no debería exceder las 250 ha, pues había consideraciones sociales y políticas, que se debían tomar en cuenta. En la constitución de 1993 en el artículo 88 se menciona que el estado garantiza el derecho de propiedad sobre la tierra, en forma privado o comunal o en cualquiera otra forma Asociativa. La ley puede fijar los límites y la extensión de la tierra según las peculiaridades de cada zona (Barboza, 2013). En este contexto, en la presente investigación se encontró que el 34,3\% de los entrevistados tiene título de propiedad de sus predios, además el mayor porcentaje de ganaderos con título se encuentra en el distrito de Huambo (60\%), seguido por Limabamba y Molinopampa (29\% y 27\% respectivamente); en los distritos de Florida, Yambrasbamba y Olleros (90\%; 75\% y 75\% respectivamente) se encuentran los más altos porcentajes de ganaderos que no poseen títulos de propiedad del predio, por lo que se puede afirmar que en la zona en estudio la gran mayoría de productores de ganado bovino no poseen títulos de propiedad de sus fundos; estos resultados concuerda al reporte del INEI (2012) donde a nivel nacional el 21\% de las parcelas tienen propietario con título registrado, correspondiendo al $34 \%$ de la superficie agropecuaria. La titulación de tierras en el país y en las zonas de estudio de la presente investigación son inferiores a los encontrados por (Tomás, 2011), al caracterizar las explotaciones lecheras de la Pampa Argentina donde los ganaderos son propietarios del $69,5 \%$ de la tierra, lo que facilita el desarrollo de inversiones, como indica la correlación positiva entre la superficie en propiedad y el capital operativo expresado en dólares $(\$)(\mathrm{r}=0,675 ; \mathrm{p}<0,01)$.
En el presente estudio, se encontró que las formas de acceso al hato ganadero en su mayoría es por camino de herradura y en menor porcentaje por trocha carrozable, este aspecto dificulta la competitividad de la ganadería en las zonas en estudio, ya que limita la comercialización de leche y carne por problemas de transporte, lo cual influye en calidad de estos productos debido a una serie de factores como contaminación microbiológica, modificación de los componentes fisicoquímicos, incremento de la acidez; así como también dificulta el proceso de adopción de tecnologías para el mejoramiento de la actividad ganadera.

Los pastos cultivados son la base de la alimentación de la ganadería al pastoreo ya sea a nivel de valles interandinos o en zonas alto andinas y se les considera como fuente principal para la producción ganadera; la asociación de gramíneas con leguminosas proveen un alimento completo y balanceado al ganado para la producción de leche, carne o fibras (Maicelo, 2011). En la presente investigación el 36\% cultivan pasto de corte en su mayoría en los distritos de Molinopampa, Olleros y Florida; con respecto al cultivo de pastos mejorados el $57 \%$ de los ganaderos cultivan estos pastos, por ser la principal fuente de alimentación del ganado; estos resultados son superiores a lo reportado por INEI (2012) para la región Amazonas donde el $13,57 \%$ de los productores ganaderos cultivan rye grass, también se puede afirmar que los ganaderos de la región Amazonas más del 65\% complementan la alimentación del ganado con pastos naturales, ya que también se encontró que la producción de pasto de corte en el hato ganadero en promedio es de 2,86 $\pm 6,3$ ha; la producción de pasto mejorado en promedio es de 5,10 $\pm 8,84$ ha y de pasto natural es de 4,52 $\pm 8,62$ ha, lo que demuestra que en las zonas en estudio, la gran mayoría de productores son pequeños; presentándose excepciones con algunos ganaderos que poseen mayor de 50 hectáreas que se encuentran en los distritos de Limabamba, Huambo, Yambrasbamba y Florida, siendo superior al reporte de García et al. (1990), quienes reportaron que el productor de Huancavelica posee en promedio 2.97 ha, distribuidas en 1.7 ha en alfalfa, 0.77 ha en descanso y 0.55 ha otros cultivos y al de Chalate-Molina et al. (2010), quien es su estudio reportó que en el estado de Morelos de Mexico, el 59\% de los ganaderos tiene menos de 5 ha.

\section{Cultivos agrícolas}

Según Medeiros (2009), indica que la economía agraria, especialmente la campesina se caracteriza por su heterogeneidad ya que en algunas situaciones no buscan la obtención de ganancias, sino minimizar 
sus riesgos y sobre todo la satisfacción de sus necesidades y la reproducción de su unidad, ya sea diversificando su producción y actividades económicas en las que participan o elaborando estrategias de sobrevivencia basadas en tiempo, espacio y variedad. En este contexto en la investigación se encontró que los productores de ganado bovino también se dedican al cultivo de productos agrícolas como café en Huambo, Limabamba y Yambrasbamba, papa en los distritos de Olleros y Florida; estas actividades lo realizan con la finalidad de diversificar su producción y su economía.

\section{Instalaciones ganaderas}

Según Vásquez (2016), las instalaciones y estructuras ganaderas dependen del tipo de explotación como en la intensiva, especialmente en ganado lechero, significa estabulación permanente y la explotación extensiva es la que se realiza básicamente en pastoreo y que sólo cuenta con algunas instalaciones básicas de apoyo. Los factores que influyen en el diseño de una instalación son el medio, animales, ganadero, entorno de la granja y aspectos económicos; en esta investigación los productores ganaderos en las zonas de estudio cuentan con mínimas instalaciones como manga y sala de ordeño, estas instalaciones se encuentran ubicadas en mayor porcentaje en los ganaderos de Molinopampa y Florida, que son los que más se dedican a la producción láctea; lo cual indica que más del $(80 \%)$ no poseen ninguna instalación, estas características muestran que en las zonas en estudio se realiza una explotación extensiva, lo que repercute en la productividad de la explotación, ya que al no contar con sala de ordeño influye en la calidad de la leche. Además, al tener cercos vivos para el manejo del ganado, se disminuye la temperatura en su interior y se crean microclimas que permiten tener los animales con mayor comodidad y más tranquilos para las labores que se vayan a realizar (Uribe, 2011) reflejándose en la productividad del animal. Al respecto, en el análisis realizado indica que sólo el $(12,6 \%)$ cuenta con corrales para su ganado y el $(25,9 \%)$ tiene corral de terneros.

\section{Prácticas de pastoreo}

Existen dos tipos de alambrados o cercos: eléctricos y convencionales; que influye en la decisión de la utilización de cualquiera de ellos en la raza que se maneja en la zona, si son cercos perimetrales o internos o si están sobre rutas asfaltadas y caminos vecinales, entre otras. El tipo de alambrados que se debe utilizar también puede variar de acuerdo a la actividad (cría o estabulada). Al respecto en los distritos estudiados, las prácticas de pastoreo que se tienen en su mayoría es con cerco de alambre, cercos vivos y cerco eléctrico; este es un aspecto importante a tener en cuenta en la asistencia técnica y en los proyectos ganaderos. Según el INEI (2012) en la región Amazonas los productores agropecuarios el (99.53\%) utiliza cerco de púas, el $(0.45 \%)$ utiliza cercos eléctricos y el $(0,02 \%)$ utiliza cerco de malla ganadera, estos resultados coinciden a lo encontrado en esta investigación donde la mayoría de ganaderos utilizan cerco de púas.

\section{Alimentación, registros y sanidad animal}

La alimentación es un factor importante para la producción de leche, carne, fibra y lana; representando entre el 50 al $70 \%$ de los costos de producción (Lotthammer, 2001) y además tiene una influencia del $30 \%$ en la producción y fertilidad. Como todo rumiante, los bovinos son animales forrajeros por naturaleza, esto quiere decir que las pasturas o forrajes son los alimentos con los que cubren todas sus necesidades clave (mantenimiento, crecimiento, preñez y desarrollo corporal). Los avances tecnológicos en materia de nutrición han generado nuevas formas de alimentación para los bovinos, tanto de tipo cárnico como lechero con el fin de satisfacer la siempre creciente demanda de carne y leche. Por consiguiente, los sistemas de producción bovina tienen que enfocarse sobre este aspecto fundamental del proceso. En los distritos en estudio se encontró que el 85,3\% alimenta su ganado solo con pasto y el $14,7 \%$ acude a otra fuente de alimentación además de pasto, evidenciándose más en los distritos de Molinopampa (33\%), Florida (32\%) y Olleros $(25 \%)$, por ser los que se dedican más a la explotación de leche; como se puede observar el uso de concentrado es bajo lo que repercute en la alimentación del ganado ya que el concentrado es una fuente de energía y proteína para complementar los forrajes y cumplir con las necesidades nutricionales del animal. El porcentaje de ganaderos que usan concentrado, es mucho menor a la realidad de Junín y Huancavelica, encontrándose hasta en $84.6 \%$ de los ganaderos que alimentan con concentrado (Cantaro, 2017).

Los minerales son elementos indispensables en todos los procesos vitales del organismo animal. Los minerales se encuentran formando parte de los huesos, músculos y nervios. La forma más usual de suministrar minerales a los bovinos es bajo la forma de sales mineralizadas. Estos pueden ser clasificados en macro y micro elementos (Albarracín, 2003); los ganaderos en los distritos en estudio el $97,9 \%$ 
proporciona sales minerales, de los cuales el 49,8\% lo realiza de forma semanal, el $43,2 \%$ quincenal y el $4,9 \%$ mensual; el uso de sales minerales es uno de los factores importantes en la alimentación del animal y la productividad del hato ganadero. Las empresas ganaderas necesitan dos tipos de registros en sus operaciones: Registro para costos de producción y registros de datos por vaca del hato reproductor. Los costos de producción le dicen al ganadero el nivel de rentabilidad de su establo o fundo, usualmente, este es el factor más importante que determina el éxito de la operación en el largo plazo. Los registros de costos de producción pueden responder preguntas como ¿Soy competitivo? y ¿Qué áreas de la operación necesita ser cambiadas? Decisiones sobre vacas a desechar y que vaquillas utilizar para reemplazos, son parte de las tareas diarias en la administración de una empresa. Lo ideal sería que estas decisiones se tomaran sobre la base de la rentabilidad de cada animal. Si bien no es posible medir los costos de producción para cada animal, sí es posible registrar los datos de producción, los cuales nos indican el nivel de rentabilidad de cada vaca en producción (Homero, 2011). Al respecto, este estudio indica que solamente el 18\% utilizan registros de producción, encontrándose en su mayoría en los distritos de Molinopampa, Olleros y Florida, por lo que se puede concluir que existe un alto porcentaje de ganaderos que no miden la productividad de su hato ganadero, lo cual no permite tomar decisiones acertadas con respecto a los animales a desechar y que aspectos fortalecer. Coincidiendo con Cantaro (2017), quien reportó que a nivel de Junín y Huancavelica solo 23\% tienen registros en sus hatos. Además, Avilez (2012), en Chile al caracterizar la producción bovina lechera encontró que solo, los grandes productores usan registros reproductivos asociándose esto a sus altas producciones.

La sanidad comprende una serie de medidas que se aplican a los animales para protegerlos y preservarlos de los riesgos resultantes de la entrada, radicación y propagación de plagas o enfermedades en el país, región o localidad (Castaldo, 2003). Así mismo, la sanidad animal contribuye a procurar la inocuidad de los alimentos de origen pecuario para proteger la salud de las personas de los riesgos resultantes de enfermedades transmitidas por alimentos. Es importante señalar que, si se tiene un buen programa de salud en el ganado, este repercute en un mejor comportamiento productivo, así como en la calidad de los productos que se obtienen, disminuyendo el número de animales enfermos y el costo por tratamientos. En ese contexto según el INEI (2012) reportó que en los distritos de Molinopampa y Olleros más del $87 \%$ dosifica sus animales, en los distritos de Florida y Yambrasbamba aproximadamente el $75 \%$ dosifica y en los distritos de Huambo y Limabamba el 51\% dosifica sus animales; estos datos son similares a los encontrados en esta investigación. También se encontró que solo el 32,9\% utiliza calendario sanitario, con mayor porcentaje en los distritos de Florida y Molinopampa, esto debido a que en estos distritos se dedican más a la explotación lechera.

La mastitis bovina continúa siendo considerada a nivel mundial como la enfermedad más importante de la industria lechera, debido a la disminución de la producción láctea, alteración en la calidad de la leche, costos por tratamiento y riesgo para la salud humana (Harmon, 1994; Wolter et al., 2004). En este estudio, el 31,5\% realiza descarte de mastitis, siendo mayor en los distritos de Florida y Molinopampa; en este contexto Avilez (2012), en Chile al caracterizar la producción bovina lechera encontró que en el aspecto sanitario se observa que los productores con mayor antigüedad en el rubro lechero tienen menos problemas de mastitis en su rebaño.

En relación al descarte de brucelosis y tuberculosis el $67,8 \%$ no realizan descarte de estas enfermedades, mientras que el $32,2 \%$ que realiza este descarte en su mayoría se encuentra en los distritos de Florida, Molinopampa y Olleros, al respecto Larrea (2011), al realizar un estudio sobre la caracterización y eficiencia de la producción lechera de la Pampa Argentina, encontró que, todas las explotaciones controlan anualmente la presencia de brucelosis, mientras que la tuberculosis es analizada en el 95\% de los casos, valores superiores a los encontrados en esta investigación. Con respecto a la aplicación de inyectables en este estudio, se encontró que más del $83 \%$ de ganaderos, saben aplicar inyectables.

\section{Calidad y venta de leche}

Una leche de calidad es aquella que posee una composición (grasa, proteína, lactosa, vitaminas y minerales) de excelencia, que presente bajos recuentos microbianos, libre de patógenos, sin contaminantes fisicoquímicos y con adecuada capacidad para ser procesada (Fernández et al, 2010). En este contexto en la presente investigación en los distritos en estudio el $74,8 \%$ no realiza análisis de leche, y los que realizan que son el $25,2 \%$ encontrándose en su mayoría en los distritos de Molinopampa y Olleros, ya que su principal actividad económica es la producción y comercialización de leche.

En los distritos en estudio, la mayor producción de litros de leche al día por ganadero se encuentra en 
Florida con $71,79 \pm 58,78$; seguido por Molinopampa con 44,04 $\pm 29,68$ y Olleros con 48,75 $\pm 28,39$ litros; mostrando que estos distritos son los que mayor producción de leche poseen ya que es una de sus principales actividades económicas; en los distritos de Huambo y Limabamba la producción de leche es baja, debido a que se dedican en su mayoría a la crianza de ganado bovino para carne; al respecto (Camargo y Camacho, 2000) mencionan que la interacción estrecha entre tecnología estipulada por el manejo aplicado y la productividad lechera en fincas de doble propósito es independientemente a la zona agroecológica. Los resultados de este estudio son muy superior al reporte de al reporte de Cantaro (2017), encontrando producción de leche de 5 a 10 litro día por productor, Alburez et al. (1997) reportó 7.96 1/vaca/día. Pero en general, el 95\% de ganaderos no supera la producción de leche de $100 \mathrm{~L} /$ día, tanto en zona de campiña como en jalca (Gutiérrez et al., 2010). Al respeto Larrea (2011) menciona que estas variaciones están relacionadas al medio ambiente, tipo de pasto, raza, entre otras variables. También es importante mencionar que el precio de venta del litro de leche en las zonas estudiadas, en promedio es de S/. 0,93 $\pm 0,19$ observando de acuerdo a los resultados un mejor pago en el distrito de Limabamba y menor pago en los distritos de Olleros, Yambrasbamba y Molinopampa por parte de la empresa Gloria. A nivel mundial para el año 2007el precio por cada $100 \mathrm{~kg}$ de leche fue de US\$58 (41€) (Gutiérrez et al., 2010).

El mercado de la leche en las zonas en estudio, el $8,4 \%$ es cubierto por la empresa Gloria, el $11,2 \%$ por empresas agroindustriales y un $58 \%$ por otros mercados; observando que Gloria es el principal mercado en el distrito de Olleros y en un sector de Molinopampa, en los demás distritos la venta de la leche se realiza a empresas agroindustriales y otros mercados informales.

\section{Métodos de reproducción}

La Universidad Austral de Chile (2007), encontró que en la VII región del Maule en el área de Curicó, el $75 \%$ de los ganaderos utiliza como método reproductivo monta natural, el 6\% inseminación artificial y el 19\% ambos métodos; estos resultados son diferentes a lo encontrado en este estudio donde el $67,8 \%$ utiliza monta natural, el $9.8 \%$ utiliza inseminación artificial y el 16,8\% ambos métodos y la diferencia hace transferencia de embriones, donde se puede observar que en este estudio los ganaderos adoptan más la tecnología de inseminación artificial que en la región de Chile, ya que es parecido a lo reportado por el INEI (2012) donde para la zona en estudio el 9,9\% utiliza inseminación artificial.

En la región Amazonas el método reproductivo más usado es por monta natural con muy pocos ganaderos que utilizan inseminación artificial y menos los que usan transferencia de embriones; esto debido a la falta de transferencia de tecnología, poca acceso a estos servicios y altos costos que representan. Según Lasley (1970) y Ossa (2003) el mejoramiento genético animal es el incremento en productividad y en la calidad del producto animal debido a cambios genéticos en los individuos que conforman las poblaciones ganaderas. La composición genética de los animales determina tanto: la productividad de los animales (eficiencia de los animales para transformar en producto animal los recursos forrajeros y otros insumos utilizados en su explotación), así como la calidad del producto producido por los animales (composición, sabor, valor nutricional, finura, textura, etc.) (Martínez, 2008); por consiguiente si deseamos mejorar ya sea la eficiencia de producción o la calidad del producto animal producido, debemos hacer cambios genéticos en la población animal en explotación, aumentando la frecuencia de los genes responsables de la alta productividad y de la mejor calidad. Mejoramiento ganadero en el sentido amplio de la palabra debe entenderse entonces como el arte de combinar genética y reproducción. Al respecto, Barrón (2017) menciona que por el limitado conocimiento del pequeños y medianos productores existen bajos rendimientos, notándose altas brechas productivas, tecnológicas y de infraestructura y que para mejorar estas condiciones, se requiere la participación articulada de todos los entes del subsector en base a planes concertados.

\section{Razas bovinas y categoría de ganado}

Según el INEI (2012), en los distritos en estudio el promedio de vacas por hato es de 4,50, el promedio de vaquillas es 3,0 y el promedio de vaquillonas es de 3,2 ; estos valores son similares a los encontrados en esta investigación donde el promedio de vacas por hato es de 4,50, el promedio de vaquillas es 2,88 y el promedio de vaquillonas es 3.98 ; pero inferior al reporte de Cantaro (2017), quien en su estudio de caracterización ganadea de distritos pertenecientes al departamento de Junín y Huancavelica; con número de 6,2 vacas por productor. Indicando que cada departamento del Perú presenta ganaderos con mejores o menores posibilidades adquisitivas de ganado o también relacionado a la cantidad de hectáreas de terreno que posean. Además ChalateMolina et al. (2010) reportó que el ganadero de Morelos de México tiene en promedio 45 bovinos. 
Sobre la población de ganado bovino en el hato, según INEI (2012), reporta que en los distritos de Molinopampa, Olleros, Florida y Yambrasbamba la raza que predomina es la Brown Swiss, estos datos son similares a los encontrados en este estudio, esto se debe a que en estas zonas se dedican más a la producción y comercialización de leche, INEI (2012) también reporta que en los distritos de Huambo y Limabamba predominan otras razas, a lo encontrado en esta investigación predominan la raza Simmental y sus cruces, por lo que se puede afirmar que en el Censo las otras razas están conformados por la Simmental y cruces; además, es similar al reporte de Cantaro (2017), quien encontró mayor población de vacunos Brown Swiss (82.7\%) respecto a otras razas.

\section{Valor económico del hato}

El valor económico del ganado bovino en pie, en los distritos en estudio varía según la categoría del animal, uso del animal, raza, condición corporal y otros factores, por ejemplo en este estudio se encontró que el toro mayor a 1,5 años su precio de venta en el mercado fluctúa entre 155 a 10000 soles, esta fluctuación se debe a los factores antes mencionados, ya que si está destinado para reproducción su precio es más elevado para lo cual tiene que cumplir ciertas características para ser considerado como núcleo genético apto para reproducción. También se puede observar que el valor económico de una vaca en ordeño oscila entre 400 a 7000 soles, dependiendo de su producción litros/vaca/día. El valor económico del ganado bovino en pie según razas, en los distritos en estudio se encontró que la vaca de un parto que tiene mayor precio en el mercado es de la raza Simmental con una media de S/. 3011,19 $\pm 1070,55$ seguido por la raza Holstein con una media de S/. $2288,11 \pm 388,79$ y la raza Brown Swiss con una media de S/. 2 $170,68 \pm 883.30$ soles. Los precios varían por la edad del animal, propósito (carne o leche o ambos), ejemplo las razas doble propósito se caracteriza por su tendencia a la producción de carne como de leche dado que su rusticidad las hacen adaptarse a una gran variedad de climas, estos sistemas tienen estructuras $\mathrm{u}$ organizaciones muy diversas que asociadas a la diversidad ambiental y fundamental conforman un fenómeno complejo dinámico, que sin embargo le hacen sustentables y competitivos, en comparación a los sistemas especializados (Camargo, 2000).

\section{CONCLUSIONES}

Las principales características socioeconómicas de los sistemas de producción de los distritos en estudio está dado por variables como título de propiedad del fundo el cual no poseen más del $64 \%$ de los ganaderos, acceso al hato ganadero que en su mayoría es por camino de herradura, precio del litro de leche bajo que no permiten hacer rentable al hato, precio del ganado bovino influenciado por muchos factores como categoría, raza, producción y otros; estas variables poseen un mismo factor común en todos los distritos que limita el mejoramiento de la actividad ganadera, el cual está dado por falta de asesoría técnica, transferencia y adopción de tecnologías.

El sistema de producción animal en hatos ganaderos de los distritos en estudio es semi-intensivo, con la característica que la alimentación es a base de pastos de corte, pastos mejorados y algunos productores utilizan otros alimentos terminados; Además, solamente el $18 \%$ de los ganaderos utilizan registros de producción, encontrándose en su mayoría en los distritos de Molinopampa, Olleros y Florida, traduciéndose en un alto porcentaje de ganaderos que no miden la productividad de su hato ganadero.

Las características socioeconómicas y técnicas del productor de ganado bovino de la región Amazonas influyen en la falta de aplicación de tecnologías productivas, uso inadecuado de infraestructura productiva, malas prácticas nutricionales, incorrecto uso de calendario sanitario, inadecuado uso de fármacos y la existencia de diferentes prácticas pecuarias por pisos geográficos.

\section{REFERENCIAS BIBLIOGRÁFICAS}

Albarracín, L. (2003). Manual de bovinos. Capacitación tecnológica para pequeños productores con subproductos de la caña en el departamento de Cundinamarca. Primera edición, Corpoica. Colombia. $64 \mathrm{p}$.

Alburez, C.; Saavedra, C.; Peñate, H. \& Haeussler, C. (1997). Caracterización del sistema de producción bovina de doble propósito con dos ordeños en la aldea Los Cerritos, Hiquimulilla departamento de Santa Rosa. Archivos Latinoamericanos de Producción Animal, 5(1), 656-658.

Avilez, J. (2012). Caracterización de la producción bovina lechera en la IX y X Región (Chile). Desarrollo estratégico de la producción láctea con alto contenido en ácido linoleico conjugado (CLA). Tesis doctoral, Universidad de Sevilla. España. 213 p.

Barrón, J. (2017). Plan Nacional de Desarrollo Ganadero 2017 - 2027. Lima, Perú. 41 p. 
Bolaños, O. (1999). Caracterización y tipificación de organizaciones de productores y productoras. En XI Congreso Nacional Agronómico. I Congreso Nacional de Extensión. Ministerio de Agricultura y Ganadería. Costa Rica. pp 31-39.

Camargo, M. (2000). Sistemas de vacunos doble propósito. In X Congreso Venezolano de Zootecnia. UNELLEZ-Guanare, Venezuela. pp 193-199.

Camargo, M., y Camacho, J. (2000). El forraje en el proceso de reconversión tecnológica en sistemas de doble propósito en Guanarito, Edo. Portuguesa. In Tejos, R., Zambrano C mancilla, L. y Garcia $\mathrm{W}$; eds. VI Seminario de Manejo y Utilización de Pastos y Forrajes en Sistemas de Producción Animal. Unellez, Barinas. pp. 54-71.

Cantaro, J. (2017). Caracterización de los sistemas de producción ganadera de los distritos de Nueve de Julio (Junín) y Tintay Puncu (Huancavelica) en la sierra central. Tesis de pre grado, Universidad Nacional Agraria la Molina. Lima, Perú. $111 \mathrm{p}$.

Castaldo, A. (2003). Caracterización de los sistemas de producción bovina (invernada) en el nordeste de la provincia de La Pampa (Argentina): modelos de gestión. Tesis de doctor, Universidad de Córdoba. Córdova, España. 298 p.

Chalate-Molina, H., Gallardo-López, F., PérezHernández, P., Paul Lang-Ovalle, F., Ortega-Jiménez, E., \& Vilaboa Arroniz, J. (2010). Características del sistema de producción bovinos de doble propósito en el estado de Morelos, México. Zootecnia tropical, 28(3), 329-339.

Cochran, W. (1998). Técnicas de Muestreo. Décimo Cuarta Reimpresión. Compañía Editorial Continental, S.A. de C.V. de México. México.

García, M.; Gutiérrez, G. \& Pampa, P. (1990). Caracterización de la ganadería bovina en el distrito de Ticrapo. HuancavelicaPerú. En: Anales Científicos UNALM. pp 147-162.

Gutiérrez, H., Trujillo, G., \& Martínez, M. (2010). Plan estratégico del sector ganadero bovino en el Peru (Doctoral dissertation, Tesis de maestría, PUCP. Lima Perú. $203 \mathrm{p}$.

Instituto Nacional de Estadística e Informática (INEI). (2012). IV Censo Nacional Agropecuario. Perú. [internet]. D i s p o ible e n : http://siea.minagri.gob.pe/siea/?q=ivcenso-nacional-agropecuario-2012/ivcenagro-2012

Larrea, Á. (2011). Caracterización y eficiencia de la producción lechera en el Noreste de La Pampa (Argentina). Tesis de doctor, Universidad de Córdova. Córdova, España. 183 p.

Lasley, J. (1970). Genética del mejoramiento del ganado (No. 636.0821 L375.). Unión Tipográfica Editorial HispanoAmericana. 378 p.

Martínez, R. (2008). Caracterización genética y morfológica del bovino criollo argentino de origen patagónico. Tesis de doctor, Universidad Politécnica de Valencia. Valencia, España. 244 p.

Medeiros, G. (2009). Unidad de Análisis de Políticas Sociales y Económicas. Diacnóstico sectoriales. El Sector Agropecuario. La Paz, Bolivia. 77 p.

Ossa Saraz, G. (2003). Mejoramiento genético aplicado a los sistemas de producción de carne (No. Doc. 20568), CO-BAC, Santafé de Bogotá. 140 p.

Ruiz, M., Ruiz, J., Torres, V. \& Cach, J. (2012). Estudio de sistemas de producción de carne bovina en un municipio del estado de Hidalgo, México. Revista Cubana de Ciencia Agrícola, 46(3), 261-265.

Uribe, F., Zuluaga A., Valencia L., Murgueitio E., Ochoa L. 2011. Buenas prácticas ganaderas. Manual 3, Proyecto Ganadería Colombiana Sostenible. GEF, Banco Mundial, Fedegán, Cipav, Fondo Accion, TNC. Bogotá, Colombia. $82 \mathrm{p}$.

Vásquez, H. (2016). Influencia de factores socioeconómicos en la adopción de tecnologías para el mejoramiento genético de ganado vacuno, distrito Florida, Amazonas, Perú. Tesis de maestría, Universidad Nacional Agraria la Molina, Lima, Perú. 132 p.

Maicelo, J. (2011). Manejo sustentable de pastos mejorados. UNTRM. Chachapoyas Perú. 